\title{
PAX6: a transcriptional guardian for glutamatergic fate in the developing neocortex
}

Kai Boon Tan ${ }^{1}$, Zrinko Kozic ${ }^{1,2}$, Daniel Dolboyi ${ }^{1}$, Martine Manuel 1, 2, John Mason ${ }^{1,2}$ and David Price ${ }^{1,2}$

1: Centre for Discovery Brain Sciences (CDBS), University of Edinburgh, Edinburgh, United Kingdom.

2: Simons Initiative for the Developing Brain (SIDB), University of Edinburgh, Edinburgh, United Kingdom.

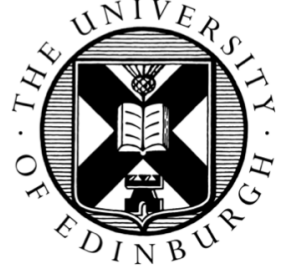

\section{Summary}

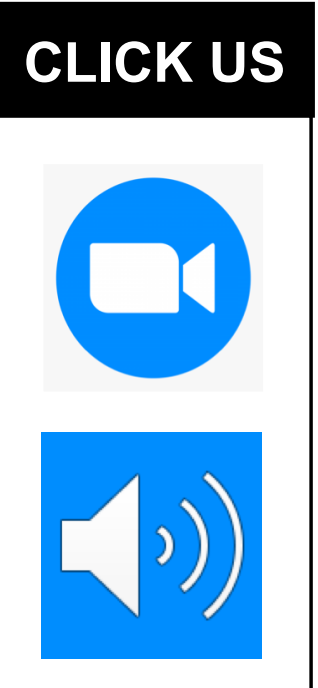

\section{SIGNIFICANCE}

- PAX6 protects glutamatergic fate in neocortical progenitors by suppressing regional morphogenetic actions

- Without PAX6, a subset of progenitors commit to multiple ectopic fates under the influences of surrounding morphogens in action

1. Summary
- PAX6 is a key transcription factor that regulates many aspects
of neurogenesis in the neocortex including glutamatergic fate
specification
- Previous studies reported neocortical progenitors commit to a
GABAergic fate upon Pax6 deletion (Stoykova et al., 2000)
- We suggest this fate change is heterogenous in nature and
region-specific depending on the morphogens in the milieu

\section{Emergence of multiple ectopic fates caused by morphogens in the absence of PAX6}

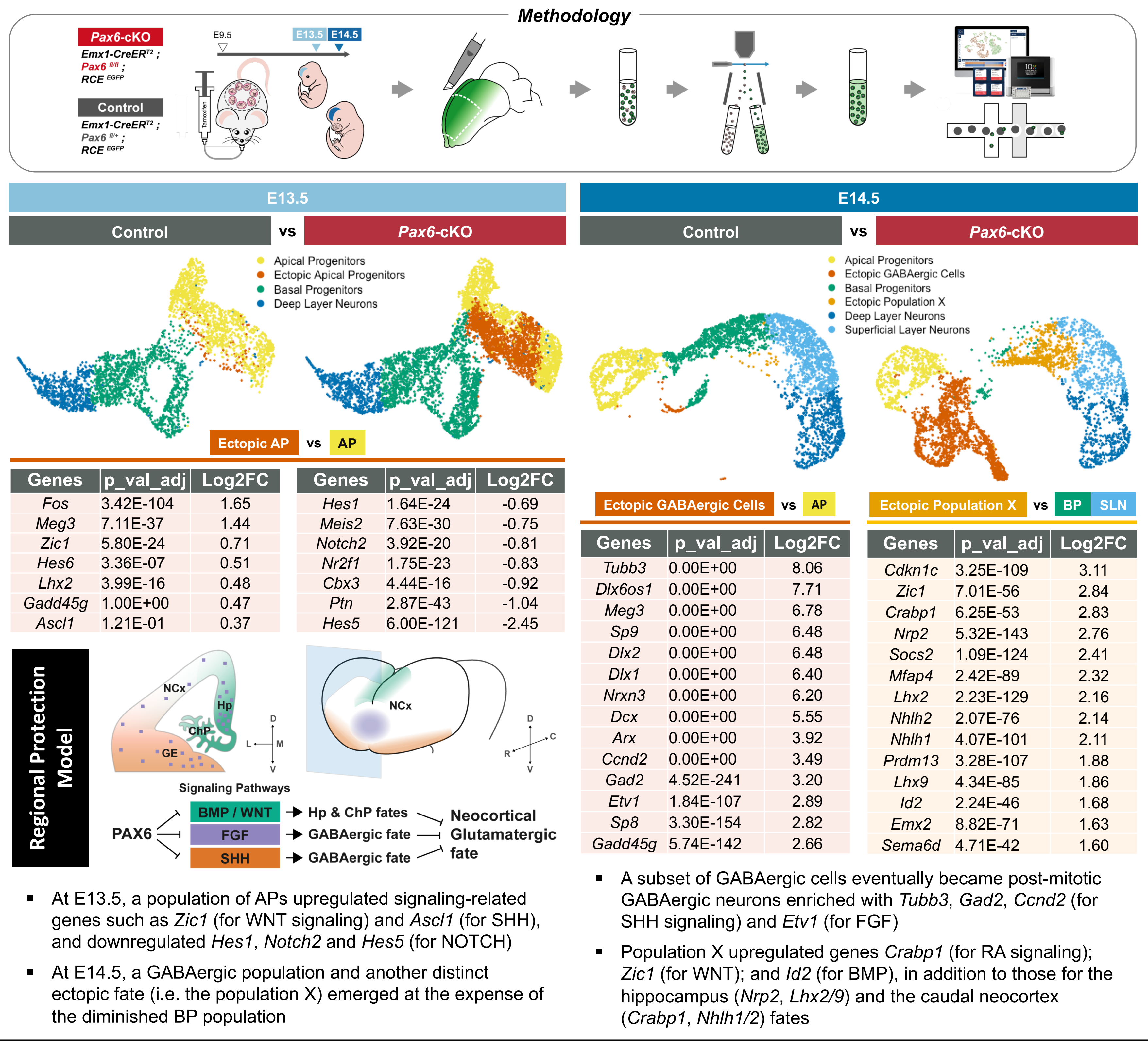

\section{Reduced GABAergic genes expression upon SHH signaling attenuation using BMP4}

E13 Pax6-cKO explant

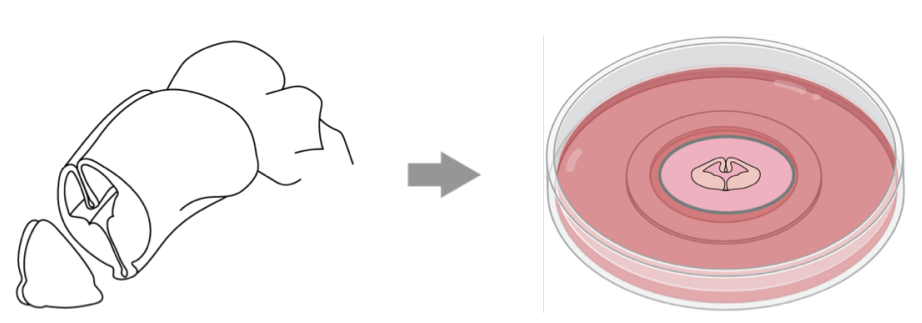

\section{Methodology}

48h BMP4 treatment Neocortex dissection

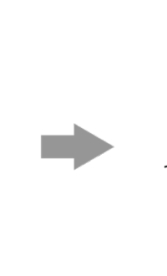

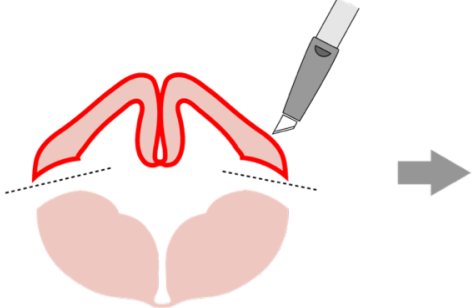

qRT-PCR quantification

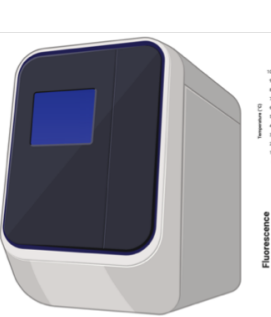

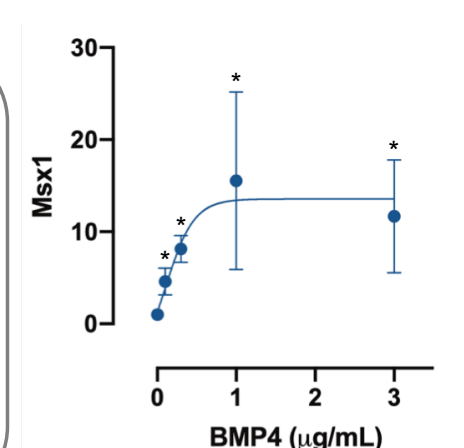
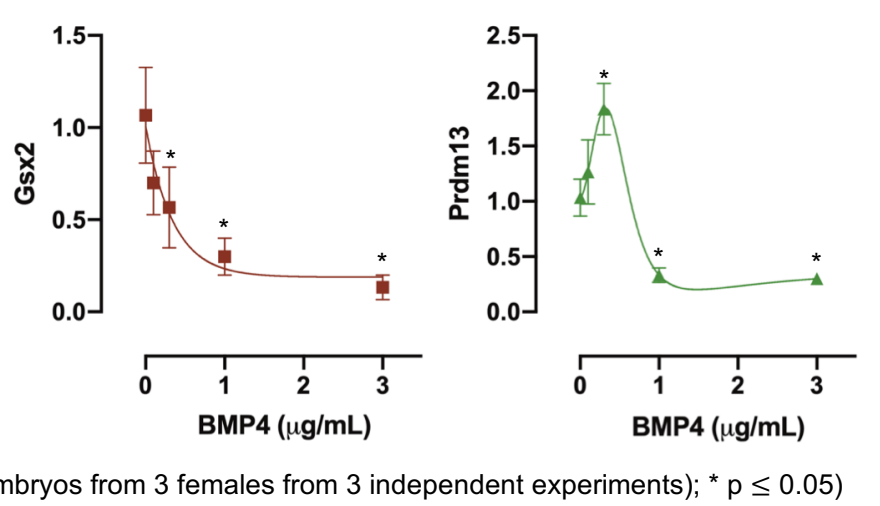